\title{
Cerebral energy metabolism in isovaleric acidaemia
}

\author{
A K Lorek, J M Penrice, E B Cady, J V Leonard, J S Wyatt, R A Iles, S P Burns, \\ E O R Reynolds
}

\begin{abstract}
A newborn infant with an acute metabolic encephalopathy caused by isovaleric acidaemia had severe impairment of cerebral energy metabolism. This was detected by phosphorus and proton magnetic resonance spectroscopy. After treatment she made excellent clinical recovery, her spectroscopic abnormalities resolved, and she was neurologically normal at the age of 1 year.

(Arch Dis Child 1996; 74: F211-F213)
\end{abstract}

Keywords: isovaleric acidaemia, magnetic resonance spectroscopy, cerebral energy metabolism.

Isovaleric acidaemia is a rare inborn error of organic acid metabolism, caused by deficiency of isovaleryl CoA dehydrogenase. Some patients present in the neonatal period with a progressive encephalopathy and non-specific symptoms such as poor feeding, weight loss, and vomiting. The characteristic odour of 'sweaty feet' may feature. The mechanism for the encephalopathy is not known, but raised concentrations of isovalerate and hyperammonaemia are likely to be important. The effect of these may be mediated through disordered mitochondrial metabolism, so we assessed cerebral metabolism before and after treatment in an acutely sick neonate with isovaleric acidaemia using phosphorus $\left({ }^{31} \mathrm{P}\right)$ and proton $\left({ }^{1} \mathrm{H}\right)$ magnetic resonance spectroscopy

Paediatrics, University College London School of Medicine, London

A K Lorek

J Penrice

J S Wyatt

E O R Reynolds

Department of

Medical Physics and

Bioengineering,

University College

Hospitals NHS Trust

London

E B Cady

Metabolic Unit, Great Ormond Street Hospital for Children

NHS Trust, London

J V Leonard

Department of Medicine, The Royal London Hospital,

London

R A Iles

S P Burns

Correspondence to: Dr Ann Lorek, The Children's Hospital Lewisham, Lewisham High Street, London SE13 6LH.

Accepted 16 November 1995

\section{Case report}

A girl was born to non-consanguineous African parents by caesarean section following prolonged rupture of the fetal membranes and induction of labour at 37 weeks of gestation. Her birthweight was $3.5 \mathrm{~kg}$. She was initially in good condition (Apgar scores of 7 at one and 10 at five minutes), but at 3 days of age, poor feeding, vomiting, and weight loss necessitated transfer to the neonatal intensive care unit. On admission she was hypotonic, hypothermic (rectal temperature $34.5^{\circ} \mathrm{C}$ ), and lethargic with signs of respiratory distress and severe dehydration. The blood glucose concentration was less than $2 \mathrm{mmol} / \mathrm{l}$ and the venous blood $\mathrm{pH}$ was $7 \cdot 18$, with a base deficit of $-15 \cdot 6$ $\mathrm{mmol} / \mathrm{l}$. She was resuscitated with intravenous glucose, albumin, and sodium bicarbonate solutions and given broad spectrum antibiotics. Intravenous calcium gluconate was administered because plasma calcium was 1.34 $\mathrm{mmol} / \mathrm{l}$ (plasma albumin $37 \mathrm{mmol} / \mathrm{l}$ ). The (MRS).

plasma ammonia was $144 \mu \mathrm{mol} / \mathrm{l}$. At about 95 hours of age, the infant became apnoeic, and required ventilation in addition to further calcium and bicarbonate. The head ultrasound appearance suggested cerebral oedema. Isovaleric acidaemia was provisionally diagnosed because of the characteristic odour, and an intravenous infusion of carnitine was started at $200 \mathrm{mg} / \mathrm{kg} / 1$ over 24 hours, to reduce intracellular isovaleryl-Co A. Subsequent gas chromatography of urine with confirmation by mass spectrometry showed a large peak of isovaleric acid $(2.4 \mathrm{mmol} / \mathrm{mmol}$ creatinine), isovaleryl glycine, and other conjugates, confirming the diagnosis.

At the age of 108 hours the first ${ }^{31} \mathrm{P}$ and ${ }^{1} \mathrm{H}$ MRS studies of the brain were performed, using a specialised transport incubator to maintain intensive support throughout the procedure. $^{1}$ The infant was subsequently transferred to Great Ormond Street Hospital for Sick Children where treatment with sodium bicarbonate, calcium, and $\alpha$-calcidol was administered, in addition to a dopamine infusion for circulatory support. After a further five days, her level of consciousness improved and the neurological abnormalities resolved. The baby was discharged home on reduced protein intake, supplemented with glucose polymer and carnitine. The second MRS studies were carried out at 18 days of age.

At 1 year of age the infant underwent a neurological examination (Amiel-Tilson) and neurodevelopmental assessment (Griffiths).

\section{MAGNETIC RESONANCE SPECTROSCOPY}

Brain spectra were acquired using a $2.4 \mathrm{~T}$ Bruker Biospec spectrometer $\left({ }^{1} \mathrm{H}\right.$ frequency $100.3 \mathrm{MHz},{ }^{31} \mathrm{P} 40.6 \mathrm{MHz}$ ) equipped with actively shielded gradient coils. For the first study, an inductively coupled, series tuned, ${ }^{1} \mathrm{H}$ Helmholtz transmitter coil of $18 \mathrm{~cm}$ in diameter was used in conjunction with a sepaand ${ }^{1} \mathrm{H}$ ) surface receiver coil of $10 \mathrm{~cm}$ in diameter. For the second study, a specially designed double tuned $\left({ }^{31} \mathrm{P}\right.$ and $\left.{ }^{1} \mathrm{H}\right)$ Helmholtz coil of $15 \mathrm{~cm}$ in diameter was used. ${ }^{2}$ The head was positioned supine and studies were performed without sedation.

During the first ${ }^{31} \mathrm{P}$ study, data were acquired from the occipital region of the brain using the surface coil. Acquisition conditions were: single pulse $\left(90^{\circ}\right.$ coil centre flip angle); recovery time (TR) 20 seconds; 64 averaged free induction decays. For all the other studies, ${ }^{1} \mathrm{H}$ and ${ }^{31} \mathrm{P}$ spectra were acquired using the rate, inductively coupled, double tuned $\left({ }^{31} \mathrm{P}\right.$ 

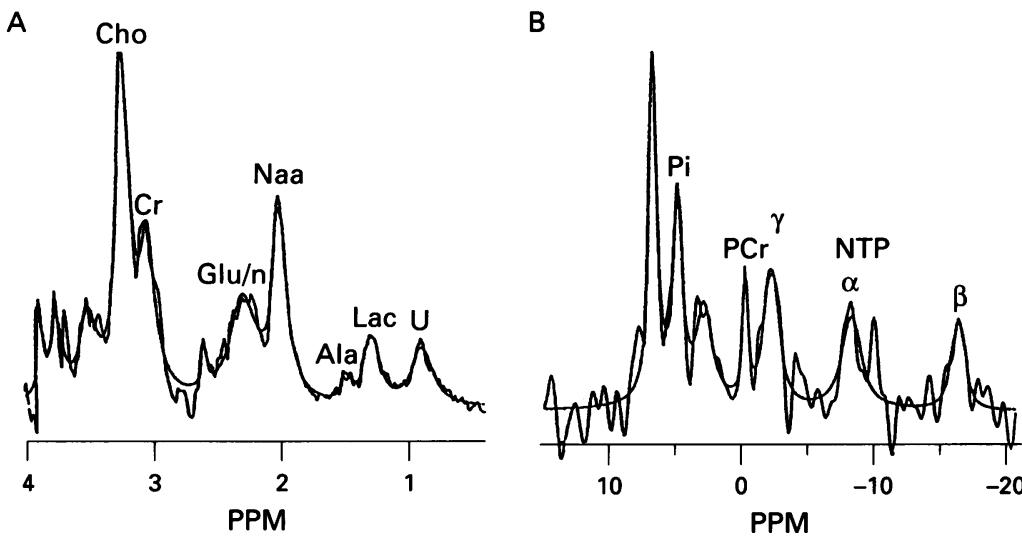

Figure $1{ }^{1} H(A)$ and ${ }^{31} P(B)$ in vivo spectra acquired at 108 hours of age. The ${ }^{1} H$ spectrum shows a high lactate peak in addition to abnormal resonances at about $1.5 \mathrm{ppm}$ and at $0.9 \mathrm{ppm}$ (attributed to alanine and isovaleric acid, respectively). The ${ }^{31} \mathrm{P}$ spectrum indicates a low $[\mathrm{PCr}]:[\mathrm{Pi}]$ suggesting impaired cerebral oxidative phosphorylation.

point resolved spectroscopy (PRESS) localisation technique. ${ }^{2}{ }^{3}{ }^{1} \mathrm{H}$ PRESS spectra were collected from an $8 \mathrm{ml}$ cubic volume of interest (VOI) positioned in the thalamus with the aid of a sagittal scout magnetic resonance image coplanar with the mid line. ${ }^{1} \mathrm{H}$ PRESS acquisition conditions were: echo time (TE) $270 \mathrm{~ms}$; TR $1730 \mathrm{~ms}$, with 256 echoes averaged. ${ }^{31} \mathrm{P}$ PRESS acquisition conditions were: $4 \times 5 \times 5$ $\mathrm{cm}^{3}$ central VOI; TR 2 seconds; TE $12 \mathrm{~ms}$, with 512 echoes averaged. Pulse amplitudes for ${ }^{31} \mathrm{P}$ were scaled from those optimised for ${ }^{1} \mathrm{H}$ using a previous calibration. ${ }^{2}$ All spectra were analysed by Lorentzian fitting in the frequency domain using $\chi^{2}$ minimisation with a gradient-search technique and prior knowledge. ${ }^{4}$

\section{Results}

Figure 1A shows the brain ${ }^{1} \mathrm{H}$ spectrum obtained at 108 hours of age. In addition to the detection of major peaks due to choline containing compounds (cho), creatine $(\mathrm{Cr}$; including phosphocreatine (PCr)), and $\mathrm{N}$ acetyl aspartate (NAA), several abnormalities were present.

The broad peak at $2.3 \mathrm{ppm}$, due mainly to glutamate and glutamine, had a peak area $1 \cdot 4$ times that of (NAA) at about $2 \cdot 0 \mathrm{ppm}$. This ratio was more than five standard deviations
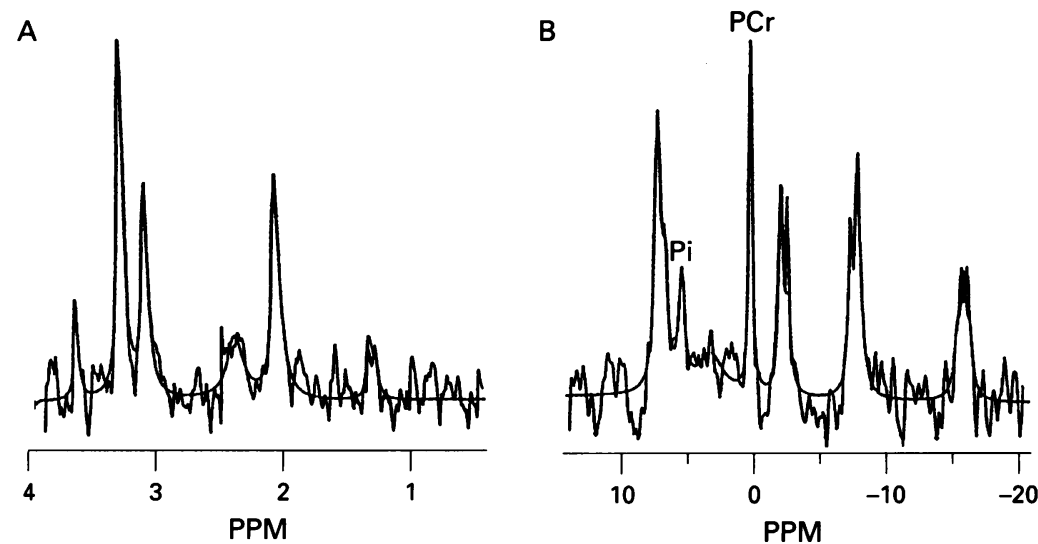

Fig $2{ }^{1} \mathrm{H}$ CSF spectrum at 96 hours. Isovalerate (IV) is detected at $0.9 \mathrm{ppm}$. Acetoacetate $(A A)$ and acetone $(A)$ are seen at $2 \cdot 2-2 \cdot 3 \mathrm{ppm}$, and 3-hydroxybutyrate (HB) at $1.2 \mathrm{ppm}$. above the mean of age matched controls. The lactate:NAA peak area ratio $(0.5)$ was also raised compared with age matched controls. ${ }^{5}$ The peak at $0.9 \mathrm{ppm}(\mathrm{U})$ was possibly attributable to isovaleric acid, and a peak at about $1.5 \mathrm{ppm}$ was probably alanine. High resolution ${ }^{1} \mathrm{H}$ MRS of cerebrospinal fluid (CSF) taken at 96 hours of age (fig 2) showed raised isovalerate concentrations at $0.9 \mathrm{ppm}$, confirming the in vivo assignment, as well as acetone and acetoacetate $(2 \cdot 2-2 \cdot 3 \mathrm{ppm})$, and 3-hydroxybutyrate $(1.2 \mathrm{ppm})$. Inositol and glucose were also present. The ${ }^{31} \mathrm{P}$ spectrum acquired invivo at 108 hours of age (fig $1 \mathrm{~B}$ ) revealed a major impairment of oxidative phosphorylation, as judged by the severely reduced [PCr]:[inorganic phosphate $(\mathrm{Pi})$ ] ratio of 0.37 (control data have $95 \%$ confidence limits 0.70 to $1 \cdot 36) .^{5}$

In the second cerebral MRS study, performed at 18 days of age, both ${ }^{1} \mathrm{H}$ and ${ }^{31} \mathrm{P}$ spectra had returned to normal (figs $3 \mathrm{~A}$ and B); the lactate:NAA peak area ratio had fallen to 0.22 and the [PCr]:[Pi] was $1 \cdot 72$, indicating normal cerebral energy metabolism. Intracellular $\mathrm{pH}$ remained normal; it was $7 \cdot 14$ at 108 hours and $7 \cdot 18$ at 18 days.

At 1 year of age neurological assessment was normal, and the Griffiths quotient was, overall, within the normal range, but with some subscore variability.

\section{Discussion}

We have demonstrated profound abnormalities of cerebral metabolism in a newborn infant with acute encephalopathy due to isovaleric acidaemia. The acute metabolic encephalopathy is likely to be multifactorial ${ }^{7}$; in this case the infant was initially hypoglycaemic and acidotic with a mildly raised ammonia, as well

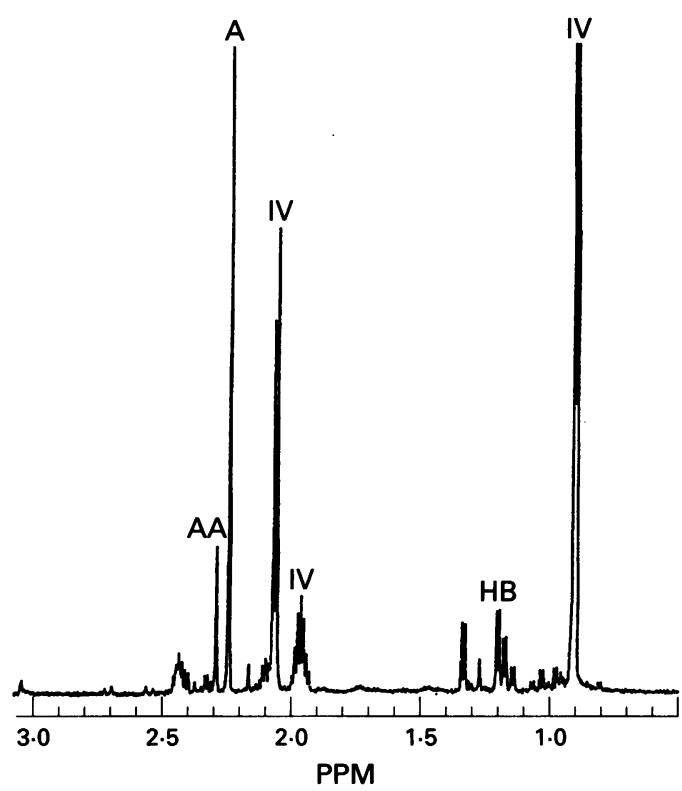

Figure $3{ }^{1} H(A)$ and ${ }^{31} P(B)$ in vivo spectra obtained at 18 days of age. The abnormal peaks seen in the ${ }^{1} H$ spectrum at age 108 hours are no longer detectable; lactate: NAA is now normal. In the ${ }^{31} P$ spectrum $[P C r]:[P i]$ has now become normal. These observations indicate that both glycolysis and oxidative phosphorylation are functioning normally. 
as having raised isovaleric acid and probable cerebral oedema. The encephalopathy persisted despite correction of systemic acidosis and hypoglycaemia. A reduced [PCr]:[Pi] ratio at age 108 hours indicated impaired oxidative phosphorylation. The raised alanine may reflect protein catabolism or be secondary to the lactic acidosis. The increased lactate:NAA peak area ratio could be consistent with a compensatory increase in anaerobic glycolysis, but could also be associated with reduced NAA due to neuronal loss. ${ }^{8}$ Normal progress of the infant at age 1 year would suggest the former. The increased glutamate and glutamine peak at about $2.03 \mathrm{ppm}$ is likely to be a consequence of increased ammonia and may have contributed to the encephalopathy. ${ }^{9}$ However, acetone and acetoacetate resonances, which were observed in the CSF, may have contributed to these peaks. The normal spectrum at 18 days of age contrasts with metabolic abnormalities following neonatal hypoxicischaemic injury in which there are reports of an increase in cerebral lactate at 7 days, ${ }^{10}$ and reduced NAA concentrations at 13 days. ${ }^{11}$ In the only other MRS study of a child with isovaleric acidaemia, a well infant aged 5 months was studied by ${ }^{1} \mathrm{H}$ MRS and found to have normal spectra. ${ }^{12}$

Although two different techniques were used to obtain the ${ }^{31} \mathrm{P}$ spectra, data from normal controls were available for both methods. The ${ }^{31} \mathrm{P}$ surface coil spectrum at 108 hours was deemed to be very abnormal on the basis of low $[\mathrm{PCr}]:[\mathrm{Pi}]$ when compared with data acquired in extensive previous studies. ${ }^{6}{ }^{31} \mathrm{P}$ PRESS results from five normal infants (GPA range 34 to 40 weeks) give a [PCr]:[Pi] of 1.59 (SD 0.37 ) (Cady EB, unpublished data), implying that the impairment of energy metabolism had resolved by 18 days of age. We have already seen catastrophic failure of cerebral energy generation and subsequent death in two infants; one with argininosuccinic aciduria and one with propionic acidaemia. ${ }^{13}$ However, in the infant described here the spectroscopic abnormalities resolved two weeks after the start of treatment, suggesting that no permanent injury had occurred. This study shows that in isovaleric acidaemia, while many factors may contribute to the encephalopathy, these are potentially reversible, provided that the production and accumulation of toxic metabolites can be attenuated - for example, by removal of isovalerate as isovalerylcarnitine - and that measures are taken to protect cerebral perfusion and provide substrate for energy production early in the encephalopathy. Thus damage caused by release of excitatory neurotransmitters (with consequent cellular damage and programmed cell death) will not be initiated. These observations also show the potential of ${ }^{31} \mathrm{P}$ and ${ }^{1} \mathrm{H}$ MRS in acute neonatal encephalopathy to investigate abnormal cerebral metabolism, as well as provide diagnostic information. MRS could also be used to monitor response to treatment, and hence help determine optimal treatment of some inborn errors of metabolism.

We thank Charlotte Bennett, Ann Stewart, Jan Townsend, Phillip Amess and Anne Seymore.

1 Chu A, Delpy DT, Thalayasingham S. A transport and life support system for newborn infants during NMR spectroscopy. In: Rolfe P, ed. Fetal and neonatal physiological measurements. London: Butterworths, 1986: 409-15.

2 Cady EB. Quantitative combined phosphorus and proton PRESS of the brains of newborn human infants. Magnet Reson Med 1995; 33: 557-63.

3 Bottomley PA. Spatial localization in NMR spectroscopy in vivo. Ann NY Acad Sci 1987; 508: 333-48.

4 Cady EB. A reappraisal of the absolute concentrations of phosphorylated metabolites in the human neonatal cerebral cortex obtained by fitting Lorentzian curves to the ${ }^{31} \mathrm{P}$ NMR spectrum. f Magnet Reson 1991; 91: the ${ }^{31} \mathrm{P}$.

5 Cady EB, Lorek A, Penrice J, Aldridge R, Wylezinska M, Wyatt JS, et al. ${ }^{1} \mathrm{H}$ magnetic resonance spectroscopy of the brains of normal infants and after perinatal hypoxiaschaemia. Magnet Reson Mat Physics Biol Med 1994; 2: 353-5

6 Azzopardi D, Wyatt JS, Hamilton PA, Cady EB, Delpy DT, Hope PL, et al. Phosphorus metabolites and intracellular $\mathrm{pH}$ in the brains of normal and small for gestational age nfants investigated by magnetic resonance spectroscopy. Ped Res 1989; 25: 440-4.

7 Surtees RJ, Leonard JV. Acute metabolic encephalopathy. $\mathcal{f}$ Inherit Metab Dis Suppl 1989; 1: 42-54.

8 Miller BL. A review of chemical issues in ${ }^{1} \mathrm{H}$ NMR spectroscopy; N-Acetyl-aspartate, creatine and choline. NMR Biomed 1991; 4: 47-52.

9 Connelly A, Cross JH, Gadian DG, Hunter JV, Kirkham FJ, Leonard JV. Magnetic resonance spectroscopy shows increased brain glutamine in ornithine carbamoyl transferase deficiency. Ped Res 1993; 33: 77-81.

10 Groendaal F, Veenhoven RH, Van der Erand J, Jansen GH Witkamp TD, de Vries LS. Cerebral lactate and N-acetylaspartate/choline ratios in asphyxiated full-term neonates demonstrated in vivo using proton magnetic resonance spectroscopy. Ped Res 1994; 35: 148-51.

11 Peden CJ, Rutherford MA, Sargentoni J, Cox J, Bryant DJ, Dubowitz LMS. Proton spectroscopy of the neonata brain following hypoxic-ischaemic injuty. Dev Med Child Neurol 1993; 35: 502-10.

12 Gradd W, Krageloh-Mann I, Klose U, Sauter R. Metabolic and destructive brain disorders in children: Findings with ocalised proton MR spectroscopy. Radiology 1991; 181: 173-81.

13 Hope PL, Costello AM deL, Cady EB, Delpy DT, Tofts PS, Chu A, et al. Cerebral metabolism in newborn infants studied by phosphorus nuclear magnetic resonance spectroscopy. In: Rolfe P, ed. Neonatal physiological measurements. London: Butterworths, 1986: 382-9 